\title{
Oxidative Stress Markers in Early Pregnancy Loss: A Case-Control Study
}

\author{
Shahnaz Torkzahrani ${ }^{1}{ }^{\mathbb{D}}$, Padideh Janati Ataei $^{{ }^{*} \mathbb{D}}$, Mehdi Hedayati $^{2}$, Soheila Khodakarim ${ }^{3}$, Zohre \\ Sheikhan1, Moniresadat Khoramabadi ${ }^{1}$, Akramsadat Sadraei ${ }^{4}$
}

\begin{abstract}
Objectives: Evidence suggests that oxidative stress (OS) plays a prominent role in the pathophysiology of pregnancy complications in women. The present study was conducted to determine the levels of OS markers in early pregnancy loss and to compare the results with those in healthy pregnant women.

Materials and Methods: A total of 32 women with early pregnancy loss and 32 healthy women in the first trimester of pregnancy, with similar demographic characteristics entered this study as the cases and controls. Serum levels of malondialdehyde (MDA), total antioxidant capacity (TAC), uric acid, and bilirubin levels were determined in both groups. The data obtained were then analyzed and compared between the groups using the independent samples t test and Mann-Whitney U test.

Results: The 2 groups matched in terms of personal-demographic characteristics including mother's age, father's age, gravidity, and body mass index (BMI). MDA levels increased significantly in the women with spontaneous abortion compared to the healthy pregnant women $(4.35 \pm 1.47$ vs. $3.42 \pm 1.68 \mu \mathrm{M} / \mathrm{L} ; P=0.026)$ and TAC decreased significantly in the cases compared to the healthy controls (552.34 \pm 212.79 vs. $1003.23 \pm 1168.68 \mathrm{U} / \mathrm{mL} ; P=0.040)$. Uric acid and bilirubin levels did not differ between the groups. Conclusions: The results of this study provides further evidence on the effect of increased OS on the incidence of early spontaneous abortion in the first trimester of pregnancy. High serum MDA levels and low TAC during pregnancy were 2 risk factors for spontaneous abortion. The present findings support the hypothesis that OS plays a key role in the etiopathogenesis of spontaneous abortion. Further studies are required for assessing the preventive role of antioxidant therapy in this complication.

Keywords: Oxidative stress, Pregnancy, Spontaneous abortion
\end{abstract}

\section{Introduction}

Spontaneous abortion is the most frequent pregnancy complication affecting $50 \%$ of all fertilization and $12 \%$ $15 \%$ of clinical pregnancies (1). More than $80 \%$ of spontaneous abortions occur in the first trimester of pregnancies (2) and the risk of abortion declines with increasing gestational age (3).

Women who have experienced spontaneous abortion are faced with more complications in their next pregnancy, including the threat of another pregnancy loss, preterm birth, small for gestational age (SGA), and postpartum hemorrhage (4). Following abortion, changes occur in the physiology of the body, lifestyle, and genital health that may have long-term effects on the physical, mental, and social health of women. Abortion is a tragic event that is followed by stress and depression in some mothers (5). The risk of major depression in women who have had an abortion increases two-fold and it often occurs during the first week after abortion (6). Although abortions rarely cause severe maternal complications, they impose a heavy burden due to the high costs of diagnostic tests, hospitalization, surgery, and treatment follow-up. A report states that the mortality rate due to the abortion is 0.05-0.22 per 100000 maternal mortalities in the United Kingdom (7).

The etiology of spontaneous abortion is multi-factorial, including genetic, endocrine, anatomical, immunological, microbiological, behavioral, and environmental factors $(2,8,9)$. However, the etiopathogenesis of $30 \%-50 \%$ of abortions is unknown (10).

There are many possible reasons for spontaneous abortion, from which oxidative stress (OS) could be a major one. Several studies have examined the role of OS in the incidence of pregnancy complications such as recurrent abortion (11), intrauterine growth restriction or IUGR (12), preterm birth (13), preeclampsia $(14,15)$, and gestational diabetes (16-17), most of which have used malondialdehyde (MDA) as a clinical marker of OS.

Antioxidants prevent the overproduction of reactive oxygen species (ROS) under normal conditions; however, the high production of ROS may lead to an unfavorable condition for natural conception in women by defeating 
the body's natural antioxidant defense system (18), which then leads to OS (19). OS affects the entire reproductive period and plays an important role in normal pregnancy.

During pregnancy, OS occurs in response to increased fetoplacental energy demands. In the first trimester, trophoblastic tissue is damaged for being at a sparing level, low activity of the main antioxidants, and oxygen sensitivity. Therefore, pregnancy loss would occur if this damage cannot be controlled (20).

Although ultrasound tests and serial beta-human chorionic gonadotropin (B-HCG) measurements are partly enable to diagnose the abortion, only a few sporadic studies have been conducted on the biomarkers that can help to detect women at risk for spontaneous abortion. There are no definitive methods of measurement that can yield a reliable estimate of OS; therefore, evaluating a series of markers may be the optimal solution.

This study aimed to evaluate the status of OS in spontaneous abortion by measuring some of its biomarkers to detect women at risk of abortion and to promote their reproductive health.

\section{Materials and Methods}

Study Participants

A multicenter, observational, case-control study was conducted through purposive sampling from 4 hospitals of Tehran, from January to August 2015. The study population consisted of 32 pregnant women in the first trimester with a clinically-confirmed diagnosis of spontaneous abortion as the case group and 32 normal healthy pregnant women in the first trimester as the control group.

\section{Study Design}

Inclusion criteria consisted of being in the first trimester of a singleton pregnancy, the lack of pre-pregnancy or newly-diagnosed systemic, endocrine, psychologic, or sexual diseases, not using any medications, no history of adverse life events in the past 6 months, no history of nonsteroidal anti-inflammatory drug intake and the lapse of at least one year from the previous pregnancy.

Data were collected on the subjects' age, height, prepregnancy weight, education, job, reproductive history, lifestyle, habits, and husbands' characteristics through a questionnaire.

Venous blood samples $(5 \mathrm{~mL})$ were taken from each of the subjects and their blood serum was then used to determine their MDA level, total antioxidant capacity (TAC), uric acid, and bilirubin levels at the Biochemistry Laboratory of the Cell and Molecular Endocrine Research Center, Research Institute for Endocrine Sciences, Shahid Beheshti University of Medical Sciences, Tehran, Iran, and their oxidative status was thus assessed.

Serum MDA level was determined by the MDA Assay Kit using thiobarbituric acid (TBA) method (TBARS assay kit, ZellBio, GmbH, Germany). The assay precision (CV4.3\%) and sensitivity were $4.4 \%$ and $0.1 \mu \mathrm{M}$, respectively.

Likewise, serum TAC was determined by the TAC Assay Kit (TAC assay kit, ZellBio, GmbH, Germany). The assay precision (CV6.7\%) and sensitivity were $4.6 \%$ and 100 $\mu \mathrm{M}$, respectively.

Moreover, the serum uric acid level was determined by uric acid assay kit using enzymatic method (Uric Acid assay kit, Padginteb Co. Tehran, Iran). The assay coefficient of variation percentage and sensitivity were $2.1 \%$ and $0.3 \mathrm{mg} / \mathrm{dL}$, respectively.

Furthermore, the serum total bilirubin level was determined by bilirubin assay kit using chemical diazotization method (Bilirubin assay kit, Padginteb Co. Tehran, Iran). The assay coefficient of variation percentage and sensitivity were $2.1 \%$ and $0.07 \mathrm{mg} / \mathrm{dL}$, respectively.

\section{Statistical Analyses}

The data obtained were analyzed in SPSS version 21.0 for Windows. Data were reported as mean \pm standard deviation (SD). The normality of the collected data was assessed using the Kolmogorov-Smirnov test and the differences between the groups were analyzed using the independent samples $t$ test or Mann-Whitney U test. The chi-square test was used to compare the groups in terms of the categorical variables and the two-tailed $t$ test to compare them in terms of the continuous variables. The results were presented in the form of statistical tables and figures. The level of statistical significance was set at $P$ value $<0.05$.

\section{Results}

The assessment of demographic characteristics showed that the mean \pm SD of mother's age in the abortion group was $28.68 \pm 6.347$ and in the normal healthy pregnancy group was $28.96 \pm 5.349$ years $(P=0.849)$. There were no significant differences between 2 groups in terms of father's age, duration of marriage, the interval between pregnancies, gestational age, number of pregnancies, body mass index (BMI), folic acid intake before pregnancy, familial history of abortion, exposure to second hand smoke and fever in pregnancy $(P>0.05)$ (Table 1$)$.

The minimum and maximum serum MDA levels in the case group were 1.62 and $6.29 \mu \mathrm{M}$, respectively. The minimum and maximum serum MDA levels in the control group were 1.12 and $6.25 \mu \mathrm{M}$, respectively.

Likewise, the minimum and maximum serum TAC in the case group were 114 and $1290 \mu \mathrm{M}$, respectively, while they were 157 and $6336 \mu \mathrm{M}$, respectively, in the control group.

Furthermore, serum MDA level in the abortion group was higher than that in the normal pregnancy group $(P=0.026$; Table 2; Figure 1$)$, and serum TAC in the case group was lower than that in in the control group $(P=0.040$; Table 2; Figure 2).

There was no significant difference between 2 groups in terms of serum uric acid levels $(3.9639 \pm 1.0008 \mathrm{mg} /$ 
Table 1. Demographic Characteristics of Participants

\begin{tabular}{|c|c|c|c|}
\hline Characteristic & Case Group (n=32) & Control Group ( $n=32)$ & P Value \\
\hline Mother's age $(y)^{a}$ & $28.68(6.34)$ & $28.96(5.34)$ & $0.849^{*}$ \\
\hline Father's age $(y)^{a}$ & $34.46(6.75)$ & $34.15(5.00)$ & $0.834^{*}$ \\
\hline Marital duration $(y)^{a}$ & $7.15(5.39)$ & $7.65(5.15)$ & $0.706^{* *}$ \\
\hline Birth Interval $(y)^{a}$ & $3.29(4.25)$ & $3.87(3.63)$ & $0.561^{* *}$ \\
\hline Gestational age $(w k)^{a}$ & $9.75(2.83)$ & $11.71(2.26)$ & $0.06^{* *}$ \\
\hline Gravidity $^{a}$ & $1.75(0.80)$ & $2.09(0.97)$ & $0.129^{* *}$ \\
\hline $\mathrm{BMI}\left(\mathrm{kg} / \mathrm{m}^{2}\right)^{\mathrm{a}}$ & $25.92(4.71)$ & $28.02(5.87)$ & $1.119^{*}$ \\
\hline Folic acid before pregnancy ${ }^{b}$ & 15 (46.9) & $12(37.5)$ & $0.448^{* * *}$ \\
\hline Familial history of abortion ${ }^{\mathrm{b}}$ & $11(34.4)$ & 7 (21.9) & $0.266^{* * *}$ \\
\hline Secondhand smoke ${ }^{b}$ & $\wedge(25)$ & $12(37.5)$ & $0.281^{* * *}$ \\
\hline Fever in pregnancy ${ }^{b}$ & $5(15.6)$ & $3(9.4)$ & $0.450^{* * *}$ \\
\hline
\end{tabular}

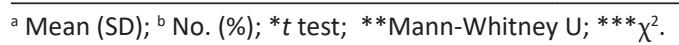

Table 2. A Comparison of Mean Serum Levels of Oxidative Stress Biomarkers Between the Case and Control Groups

\begin{tabular}{lccc}
\hline OS Biomarker & Case Group $(\mathbf{n}=\mathbf{3 2})$ & Control Group $(\mathbf{n}=\mathbf{3 2})$ & $\boldsymbol{P}$ Value \\
MDA $(\mu \mathrm{M})$ & $4.3517 \pm 1.4711$ & $3.4222 \pm 1.6821$ & 0.0267 \\
$\operatorname{TAC}(\mu \mathrm{M})$ & $552.3462 \pm 212.7925$ & $1003.2308 \pm 1168.6865$ & 0.0409 \\
\hline
\end{tabular}

OS: oxidative stress; MDA: malondialdehyde; TAC: total antioxidant capacity.

dL vs. $3.5550 \pm 0.9854 \mathrm{mg} / \mathrm{dL})(P=0.107)$. In addition, no significant difference was observed between the abortion group and healthy pregnancy group in terms of serum bilirubin levels $(1.0358 \pm 0.4309 \mathrm{mg} / \mathrm{dL}$ vs. $0.8422 \pm 0.4789$ $\mathrm{mg} / \mathrm{dL})(P=0.097)$.

\section{Discussion}

This study is one of the very few published studies to determine the levels of OS markers in miscarriage and is the first effort made in Iran assessing OS biomarkers among pregnant women lived in Tehran.

This study intended to evaluate OS status in the women with spontaneous abortion and those with normal pregnancy currently in the first trimester by measuring serum levels of OS biomarkers. The results showed a significant increase in serum levels of MDA and a significant reduction in serum TAC in the cases compared with the controls; however, the 2 groups did not differ significantly in terms of serum uric acid and bilirubin

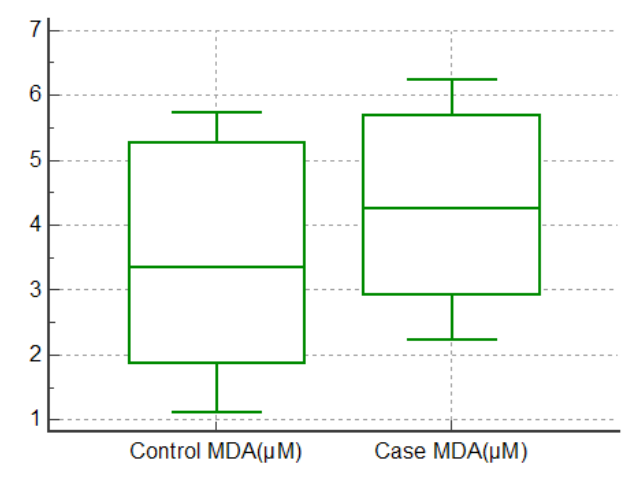

Figure 1. A Comparison of Serum MDA Levels Between the Case and Control Groups. Abbreviations: MDA, malondialdehyde. levels. The present findings are consistent with the results obtained by Issa et al in assessing OS status in the women with spontaneous abortion. Their study revealed higher serum MDA levels in the women who had experienced an abortion compared to the healthy non-pregnant women (21). Ozkaya et al showed serum MDA levels were higher in the women with vaginal hemorrhage and spontaneous abortion compared with healthy pregnant women at the same gestational age (22).

MDA has been regarded as one of the most useful markers of OS in many studies which causes some pregnancy complications. Sutama et al reported higher serum levels of MDA in the pregnant women at risk of abortion than that in the women with normal pregnancy, and proposed increased MDA levels as a potential risk factor for threatening abortion (23).

OS affects the entire reproductive period in a woman's life and plays an important role in the women's fertility by interfering with the pathophysiology of complications such as preeclampsia, hydatidiform mole, and abortion (24). Increased ROS may lead to recurrent abortions by causing double-strand DNA breaks in the sperm and oocyte. In addition, the study of Sumitha et al showed increased levels of OS in the women with recurrent abortions (11). Moreover, the study performed by Ghneim et al revealed that OS could be a major causative factor of recurrent abortion (25).

Daglar et al found that serum MDA levels in the women with abortion at the first trimester were slightly higher than that in the women with normal pregnancy; although the difference was not statistically significant $(P=0.083)$. Furthermore, women with abortion had a higher glutathione peroxidase (GPX) level compared to those with normal pregnancy. This study could not find a 


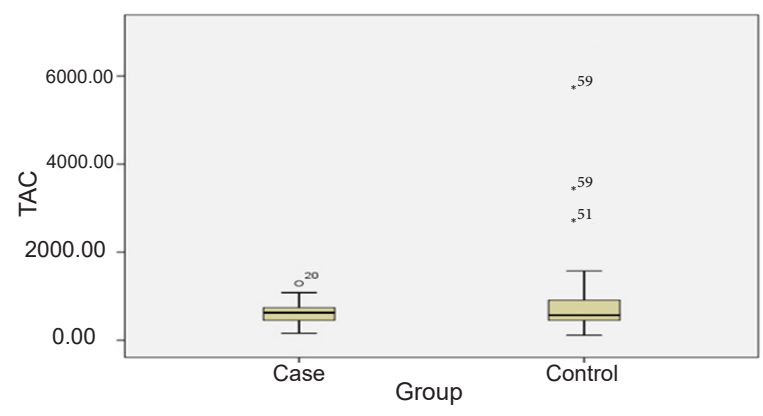

Figure 2. Total Antioxidant Capacity (TAC) in the Case and Control Groups.

significant increase in MDA levels in the abortion group maybe because of the increased activity of antioxidant defense system, which drives the oxidant-antioxidant balance toward the increasing antioxidant defense and which can be justified by the high GPX levels in the abortion group (26).

Direct measurement of free radicals is very difficult due to their instability and it is possible through the measurement of lipid peroxidation products such as MDA. Analysis of MDA means the indirect measurement of free radicals and MDA can therefore help determine free radicals in pathologic conditions (27). MDA is widely used today as a clinical marker of lipid peroxidation and is much more accurate and stable than other markers (28-29). Another study demonstrated that increased lipid peroxidation in the form of increased MDA level, decreased superoxide dismutase level, and altered thyroid profile might be involved in the termination of pregnancy and expulsion of conception products out of the uterine cavity (30).

Considering that MDA has been used as a marker for the diagnosis of OS in pathological pregnancies in many studies (11-17), the present study also examined this biomarker as a parameter of OS in the women with early spontaneous abortion.

Evidence demonstrates that lipid peroxidation increases in abortion (28) and leads to the production of MDA in the process of OS $(28,30)$, thereby justifying the increase of MDA in abortion.

Given that ROS can weaken the antioxidant system, TAC evaluation is a reasonable method for examining OS (31). Measuring TAC in the serum or plasma can provide better biological information about the individual's antioxidant status (32). In the present study, serum TAC was measured in all the participants and found to be lower in the women with abortion than that in the women with healthy pregnancy. In line with these findings, Toy et al showed a reduction in TAC in the women with abortion before the 12th week of pregnancy, which suggests the presence of OS in these women (33).

Findings of a study claimed that the total antioxidant level was significantly lower in the women who had a miscarriage (34).

Deveer et al reported a reduction in serum TAC along with vaginal hemorrhage in the pregnant women at $<10$ weeks of gestation compared to the women with normal pregnancy and argued that it is not clear whether the oxidant/antioxidant imbalance is the result or the cause of the hemorrhage experienced in the first trimester (35). To assess the antioxidant defense system and determine the presence of OS, Yiyenoğlu et al measured serum TAC in the women who had experienced recurrent abortions with unknown etiology and found that TAC was lower in the first trimester in those with recurrent abortions compared to the women with normal pregnancy, which confirms the role of OS in the etiology of recurrent abortions (36).

Serum uric acid was another marker examined in this study. The mean serum uric acid level in the abortion group was higher than that in the normal pregnancy group; however, the difference was not statistically significant. Uric acid is one of the main components of TAC, changes of which affect TAC as well. Uric acid level decreases in the first trimester of a normal pregnancy and then increases for the rest of the pregnancy (37). Clinical and experimental evidence suggests that uric acid plays a major role in the human body as an antioxidant and OS marker (38). Studies on the etiopathogenesis of abortion have not yet measured serum uric acid level as a biomarker of OS; however, the marker has been measured in some pregnancy complications such as cervical insufficiency (39) and preeclampsia (40).

In the present study, serum bilirubin level in the abortion group was higher than that in the group with normal pregnancy; however, the difference was not statistically significant. Bilirubin is a catalytic product of heme-oxygenase that inhibits lipid peroxidation, has strong antioxidant properties, and contributes to placental vessel development and the preservation of pregnancy (41). Bilirubin is also a major antioxidant component of the plasma (42). George et al reported that hypoxiainduced OS could be reduced in the placenta of rodents through the administration of bilirubin (43). Evidence also suggests that OS contributes to the development of preeclampsia, and the induction of bilirubin therefore provides a novel strategy for reducing OS in the ischemic placenta of the patients with preeclampsia due to its strong antioxidant properties (42).

\section{Strengths and Limitations}

The strengths of this study include the use of four biomarkers, including MDA, TAC, uric acid, and bilirubin, for measuring OS. All the biomarkers were measured in the same center by the same person, and all the confounding variables were controlled. Given the limited access to eligible participants, further studies are recommended to be conducted on this subject with larger sample size. 


\section{Conclusions}

This study found a significant increase in the MDA level and a significant reduction in TAC in the women with a spontaneous abortion in the first trimester of pregnancy. These parameters may indicate high levels of OS in the women with a spontaneous abortion and can therefore be helpful in the adoption of preventive approaches for the prediction or assessment of the risk of abortion, although more extensive studies are required to confirm this finding.

\section{Conflict of Interests}

Authors declare that they have no conflict of interests.

\section{Ethical Issues}

The study was approved by the Ethics Committee of Shahid Beheshti University of Medical Sciences, Iran (ethical No. 155). All participants were provided with the information regarding the study objectives in simple language. The women were included in the study after they agreed and signed the informed consent form. The ethical standards laid down in the 1964 Declaration of Helsinki were observed throughout the study and all the obtained data were documented in pre-designed data sheet.

\section{Financial Support}

Deputy of Research, Shahid Beheshti University of Medical Sciences, Tehran, Iran supported the study.

\section{References}

1. Lyu SW, Song H, Yoon JA, et al. Transcriptional profiling with a pathway-oriented analysis in the placental villi of unexplained miscarriage. Placenta. 2013;34(2):133-140. doi:10.1016/j.placenta.2012.12.003

2. 2.Cunningham FG, Spong CY, Dashe JS, Huffman BL, Casey BM, Sheffield JS. Williams Obstetrics. 24 ed. New York, NY: McGraw-Hill; 2014.

3. Juliano M, Dabulis S, Heffner A. Characteristics of women with fetal loss in symptomatic first trimester pregnancies with documented fetal cardiac activity. Ann Emerg Med. 2008;52(2):143-147. doi:10.1016/j. annemergmed.2008.03.002

4. Bhattacharya S, Townend J, Shetty A, Campbell D, Bhattacharya S. Does miscarriage in an initial pregnancy lead to adverse obstetric and perinatal outcomes in the next continuing pregnancy? Bjog. 2008;115(13):1623-1629. doi:10.1111/j.1471-0528.2008.01943.x

5. National Collaborating Centre for Ws, Children's $H$. Ectopic Pregnancy and Miscarriage: Diagnosis and Initial Management in Early Pregnancy of Ectopic Pregnancy and Miscarriage. London: National Collaborating Centre for Women's and Children's Health; 2012.

6. NeugebauerR,KlineJ,ShroutP,etal.Majordepressivedisorder in the 6 months after miscarriage. JAMA. 1997;277(5):383388. doi:10.1001/jama.1997.03540290035029

7. Cantwell R, Clutton-Brock T, Cooper G, et al. Saving Mothers' Lives: Reviewing maternal deaths to make motherhood safer: 2006-2008. The Eighth Report of the Confidential Enquiries into Maternal Deaths in the United Kingdom. BJOG. 2011;118 Suppl 1:1-203. doi:10.1111/ j.1471-0528.2010.02847.x

8. Lathi RB, Gray Hazard FK, Heerema-McKenney A, Taylor J, Chueh JT. First trimester miscarriage evaluation. Semin Reprod Med. 2011;29(6):463-469. doi:10.1055/s-0031-1293200

9. Kumar S. Occupational, environmental and lifestyle factors associated with spontaneous abortion. Reprod Sci. 2011;18(10):915-930. doi:10.1177/1933719111413298

10. Bazmi S, Behnoush B, Kiani M, Bazmi E. Comparative Study of Therapeutic Abortion Permissions in Central Clinical Department of Tehran Legal Medicine Organization before and after Approval of Law on Abortion in Iran. Iran J Pediatr. 2008;18(4):315-322.

11. Sumitha Prabhu PS, Aneesh P, Jiju JS, Reshma P, Dinesh Roy D. Evidence of increased oxidative stress and DNA damages in women with recurrent abortions. Int J Sci Eng Res. 2015;6(5):15-19.

12. Holland O, Dekker Nitert M, Gallo LA, Vejzovic M, Fisher JJ, Perkins AV. Review: Placental mitochondrial function and structure in gestational disorders. Placenta. 2017;54:29. doi:10.1016/j.placenta.2016.12.012

13. Mustafa MD, Pathak R, Ahmed T, et al. Association of glutathione S-transferase M1 and T1 gene polymorphisms and oxidative stress markers in preterm labor. Clin Biochem. 2010;43(13-14):1124-1128. doi:10.1016/j. clinbiochem.2010.06.018

14. Gupta S, Aziz N, Sekhon L, et al. Lipid peroxidation and antioxidant status in preeclampsia: a systematic review. Obstet Gynecol Surv. 2009;64(11):750-759. doi:10.1097/ OGX.0b013e3181bea0ac

15. Wu F, Tian FJ, Lin Y, Xu WM. Oxidative Stress: Placenta Function and Dysfunction. Am J Reprod Immunol. 2016;76(4):258-271. doi:10.1111/aji.12454

16. Madazli R, Tuten A, Calay Z, Uzun H, Uludag S, Ocak V. The incidence of placental abnormalities, maternal and cord plasma malondialdehyde and vascular endothelial growth factor levels in women with gestational diabetes mellitus and nondiabetic controls. Gynecol Obstet Invest. 2008;65(4):227-232. doi:10.1159/000113045

17. Lappas M, Hiden U, Desoye G, Froehlich J, Hauguel-de Mouzon S, Jawerbaum A. The role of oxidative stress in the pathophysiology of gestational diabetes mellitus. Antioxid Redox Signal. 2011;15(12):3061-3100. doi:10.1089/ ars. 2010.3765

18. Al-Gubory KH, Fowler PA, Garrel C. The roles of cellular reactive oxygen species, oxidative stress and antioxidants in pregnancy outcomes. Int $\mathrm{J}$ Biochem Cell Biol. 2010;42(10):1634-1650. doi:10.1016/j.biocel.2010.06.001

19. Agarwal A, Gupta S, Sharma RK. Role of oxidative stress in female reproduction. Reprod Biol Endocrinol. 2005;3:28. doi:10.1186/1477-7827-3-28

20. Bilici M. The Importance of Oxidative Stress in Early Week Pregnancy Losses. Crescent J Med Biol Sci. 2014;1(4):151153.

21. Issa $\mathrm{AM}$, Hassan $\mathrm{BG}$, Gatea AK. Relationship of Nitric Oxide and Malondialdehyde to Miscarriage. Med J Babylon. 2012;9(4):777-785. 
22. Ozkaya O, Sezik M, Kaya H. Serum malondialdehyde, erythrocyte glutathione peroxidase, and erythrocyte superoxide dismutase levels in women with early spontaneous abortions accompanied by vaginal bleeding. Med Sci Monit. 2008;14(1):Cr47-51.

23. Sutama GN, Surya GP. Serum Malondialdehyde Level as a Risk Factor for Threatened Abortion. Indonesian J Obstet Gynecol. 2016;3(1):11-14.

24. de Bruin JP, Dorland M, Spek ER, et al. Ultrastructure of the resting ovarian follicle pool in healthy young women. Biol Reprod. 2002;66(4):1151-1160. doi:10.1095/ biolreprod66.4.1151

25. Ghneim HK, Alshebly MM. Biochemical Markers of Oxidative Stress in Saudi Women with Recurrent Miscarriage. J Korean Med Sci. 2016;31(1):98-105. doi:10.3346/jkms.2016.31.1.98

26. Daglar K, Biberoglu E, Kirbas A, et al. The cellular immunity and oxidative stress markers in early pregnancy loss. J Matern Fetal Neonatal Med. 2016;29(11):1840-1843. doi:10.3109/14767058.2015.1064886

27. Jauniaux E, Hempstock J, Greenwold N, Burton GJ. Trophoblastic oxidative stress in relation to temporal and regional differences in maternal placental blood flow in normal and abnormal early pregnancies. Am J Pathol. 2003;162(1):115-125. doi:10.1016/s0002-9440(10)63803-5

28. Niki E. Lipid peroxidation: physiological levels and dual biological effects. Free Radic Biol Med. 2009;47(5):469-484. doi:10.1016/j.freeradbiomed.2009.05.032

29. Jauniaux E, Poston L, Burton GJ. Placental-related diseases of pregnancy: Involvement of oxidative stress and implications in human evolution. Hum Reprod Update. 2006;12(6):747-755. doi:10.1093/humupd/dml016

30. Ramandeep K, Kapil G, Harkiran K. Correlation of enhanced oxidative stress with altered thyroid profile: Probable role in spontaneous abortion. Int J Appl Basic Med Res. 2017;7(1):20-25. doi:10.4103/2229-516x.198514

31. Agarwal A, Gupta S. Role of reactive oxygen species in female reproduction and the effects of antioxidant supplementation-Part 2. Agro Food Ind $\mathrm{Hi}$ Tech. 2005;16:38-41.

32. Ghiselli A, Serafini M, Natella F, Scaccini C. Total antioxidant capacity as a tool to assess redox status: critical view and experimental data. Free Radic Biol Med. 2000;29(11):1106-
1114. doi:10.1016/S0891-5849(00)00394-4

33. Toy H, Camuzcuoglu H, Camuzcuoglu A, Celik H, Aksoy N. Decreased serum prolidase activity and increased oxidative stress in early pregnancy loss. Gynecol Obstet Invest. 2010;69(2):122-127. doi:10.1159/000262608

34. Omeljaniuk WJ, Socha K, Borawska MH, et al. Antioxidant status in women who have had a miscarriage. Adv Med Sci. 2015;60(2):329-334. doi:10.1016/j.advms.2015.06.003

35. Deveer R, Deveer M, Engin-Ustun Y, et al. Role of Oxidative Stress on Vaginal Bleeding during The First Trimester of Pregnant Women. Int J Fertil Steril. 2014;7(4):271-274.

36. Yiyenoglu OB, Ugur MG, Ozcan HC, et al. Assessment of oxidative stress markers in recurrent pregnancy loss: a prospective study. Arch Gynecol Obstet. 2014;289(6):13371340. doi:10.1007/s00404-013-3113-4

37. Toescu V, Nuttall SL, Martin U, Kendall MJ, Dunne F. Oxidative stress and normal pregnancy. Clin Endocrinol (Oxf). 2002;57(5):609-613. doi:10.1046/j.13652265.2002.01638.x

38. Menon R, Bonney E. Oxidative Stress and Preterm Birth. In: Dennery PA, Buonocore G, Saugstad OD, eds. Perinatal and Prenatal Disorders. New York, NY: Springer; 2014:95115. doi:10.1007/978-1-4939-1405-0_5

39. Zolotukhin P, Aleksandrova A, Goncharova A, Shestopalov A, Rymashevskiy A, Shkurat T. Oxidative status shifts in uterine cervical incompetence patients. Syst Biol Reprod Med. 2014;60(2):98-104. doi:10.3109/19396368.2013.8643 43

40. Sultana R, Ahmed S, Sultana N, Fazlul Karim SM, Atia F. Association of Serum Uric Acid with Preeclampsia: A Case Control Study. Delta Med Coll J. 2013;1(2):46-50. doi:10.3329/dmcj.v1i2.15918

41. Clerici G, Slavescu C, Fiengo S, et al. Oxidative stress in pathological pregnancies. J Obstet Gynaecol. 2012;32(2):124-127. doi:10.3109/01443615.2011.637139

42. George EM, Granger JP. Heme oxygenase in pregnancy and preeclampsia. Curr Opin Nephrol Hypertens. 2013;22(2):156-162. doi:10.1097/MNH.0b013e32835d19f7

43. George EM, Colson D, Dixon J, Palei AC, Granger JP. Heme Oxygenase-1 Attenuates Hypoxia-Induced sFlt1 and Oxidative Stress in Placental Villi through Its Metabolic Products CO and Bilirubin. Int J Hypertens. 2012;2012:486053. doi:10.1155/2012/486053

(C) 2018 The Author (s); This is an open-access article distributed under the terms of the Creative Commons Attribution License (http://creativecommons.org/licenses/by/4.0), which permits unrestricted use, distribution, and reproduction in any medium, provided the original work is properly cited. 